\title{
Edaravone in Amyotrophic Lateral Sclerosis-Lessons from the Clinical Development Program and the Importance of a Strategic Clinical Trial Design
}

\author{
Said R Beydoun ${ }^{1}$ and Jeffrey Rosenfeld ${ }^{2}$ \\ 1. Neuromuscular Division, Keck Medical Center, University of Southern California, LOS Angeles, CA, US; 2. Department of Neurology, Loma Linda \\ University School of Medicine, Loma Linda, CA, US
}

DOI: https://doi.org/10.17925/USN.2018.14.1.47

E daravone significantly slows progression of amyotrophic lateral sclerosis (ALS), and is the first therapy to receive approval by the Food and Drug Administration (FDA) for the disease in 22 years. Approval of edaravone has marked a new chapter in pharmaceutical development since the key trial included a novel strategic clinical design involving cohort enrichment. In addition, approval was based on clinical trials that had a relatively small patient number and were performed outside of the US. Edaravone was developed through a series of clinical trials in Japan where it was determined that a well-defined subgroup of patients was required to reveal a treatment effect within the study period. Amyotrophic lateral sclerosis is associated with wide-ranging disease heterogeneity (both within the spectrum of ALS phenotypes as well as in the rate of progression). The patient cohort enrichment strategy aimed to address this heterogeneity and should now be considered as a viable, and perhaps preferred, trial design for future studies. Future research incorporating relevant biomarkers may help to better elucidate edaravone's mechanism of action, pharmacodynamics, and subsequently ALS phenotypes that may preferentially benefit from treatment. In this review, we discuss the edaravone clinical development program, outline the strategic clinical trial design, and highlight important lessons for future trials.

\section{Keywords}

Amyotrophic lateral sclerosis, clinical trials, edaravone

Disclosures: Said R Beydoun has worked on the advisory board or as a consultant for Mitsubishi Tanabe Pharma America, as a speaker for Mitsubishi Tanabe Pharma America and received a research grant from Mallinckrodt Pharmaceuticals. Jeffrey Rosenfeld has worked on the advisory board or as a consultant for Mitsubishi Tanabe Pharma America, as a speaker for Mitsubishi Tanabe Pharma America, has received a research grant from Mallinckrodt Pharmaceuticals; and has performed research collaboration with Prosetta Biosciences.

Review Process: Double-blind peer review.

Acknowledgments: Emma East of Touch Medical Media, and Chandra Abbott of IVy Medical Communications, provided editorial assistance. Wendy Agnese, Mitsubish Tanabe Pharma America Medical Affairs, provided review for scientific accuracy.

Authorship: All named authors meet the criteria of the International Committee of Medical Journal Editors for authorship for this manuscript, take responsibility for the integrity of the work as a whole and have given final approval for the version to be published.

open Access: This article is published under the Creative Commons

Attribution Noncommercial License, which permits any non-commercial

use, distribution, adaptation and reproduction provided the original authors

and source are given appropriate credit. (C) The Authors 2018.

Received: February 202018

Accepted: March 122018

Citation: US Neurology. 2018;14(1):47-53

Corresponding Author: Jeffrey Rosenfeld, 11370 Anderson

street, Loma Linda, CA 92354, US. E.JRosenfeld@|lu edu

Support: Publication support provided by Mitsubishi Tanabe Pharma America, Inc. The authors received no funding or compensation for development and authorship of this article. The views and opinions expressed are those of the authors and are not necessarily those of Mitsubishi Tanabe Pharma America, Inc.
Motor neuron disease (MND) encompasses a range of disorders that differentially affect the upper and lower motor neurons. Amyotrophic lateral sclerosis (ALS) is the most common form of MND. In ALS, both upper and lower motor neurons are affected. ${ }^{1}$ Amyotrophic lateral sclerosis is classified as a spectrum disorder since there is great variability in the phenotypic expression of the disease. ${ }^{2}$ While there are symptomatic therapies available that may impact patient quality of life, no curative therapies have been identified. ${ }^{3,4}$ Only a small percentage of patients survive beyond 10 years, one half of patients die within 30 months of symptom onset, and $20 \%$ of patients survive between 5 and 10 years. ${ }^{1,5}$ Respiratory failure is the most common cause of mortality in patients with ALS.

There is often a delay between onset of the first symptom and establishment of the diagnosis of ALS. Time from symptom onset to diagnosis is usually up to 12 months. With the advent of new therapies, it is therefore critical to have this window shortened, before significant motor neuronal loss has accumulated. The diagnostic process is time-consuming, and a reluctance of patients to seek help, lengthy referrals, and mistaken diagnoses are all thought to contribute to the delay. ${ }^{6}$ On the part of physicians, delay in diagnosis is mainly due to lack of early recognition of the disease as symptoms may mimic other disorders that must first be ruled out. ${ }^{6}$

Familial ALS accounts for $10 \%$ of cases, with sporadic ALS accounting for $90 \%$ of cases, although many of these patients with sporadic ALS possess the same genetic abnormalities found in the familial forms. Repeat expansion in the C9orf72 gene is the most common genetic cause of familial ALS in patients of European descent, accounting for more than $30 \%$ of cases. This repeat expansion is also suggested to account for up to $10 \%$ of sporadic ALS cases. ${ }^{8}$ Over 180 different mutations in the superoxide dismutase-1 (SOD-1) gene account for a further $20 \%$ of familial ALS cases. ${ }^{5}$ 
In the US, recent registry data suggests there are around 3.9 new cases of ALS per 100,000 people each year. ${ }^{9}$ Onset of sporadic ALS usually occurs in patients in their mid-to-late 50s, however, onset of familial ALS is usually in the late teens or early adulthood. ${ }^{10}$ The clinical presentation of ALS is variable, with approximately $25 \%$ of patients presenting with bulbar-onset disease, $70 \%$ with limb-onset disease and 5\% with trunk or respiratory involvement. ${ }^{11}$ In addition, there is significant variability in the rate of disease progression, the symmetry of the disease, the degree of cognitive/behavioral symptoms (reported to occur in $20 \%$ of patients) and the extent to which upper motor neurons versus lower motor neurons are involved. Symptoms can include muscle weakness, atrophy and spontaneous twitching or fasciculations (lower motor neuron signs), hyperreflexia and hypertonia (upper motor neuron signs), dysarthria and dysphagia (bulbar-onset), and orthopnea and dyspnea (respiratory-onset). ${ }^{2,10}$

There is no single diagnostic test that confirms the diagnosis of ALS, and physicians rely on clinical examination and the El Escorial and revised Airlie House diagnostic criteria to provide a degree of diagnostic certainty. ${ }^{11}$ Electrodiagnostic studies are very valuable to confirm the diagnosis of ALS and identify the distribution of lower motor neuron abnormality. Other diagnostic studies including imaging, immunologic laboratory testing, and in some cases cerebrospinal fluid analysis and muscle biopsies are performed to exclude other disorders that may mimic the clinical signs and symptoms of ALS. ${ }^{12}$

The exact mechanisms leading to motor neuron degeneration in ALS are still unclear, however, it is thought to be due to a complex interplay between genetic and environmental factors. Several cellular and molecular mechanisms of ALS pathogenesis have been elucidated, offering some common ground among the varied phenotypic expressions of the disease. These include: glutamate-induced excitotoxicity, free radical generation, inflammation, abnormalities in nuclear-cytoplasmic transport of RNA binding proteins, mislocalization of nuclear proteins and formation of cytoplasmic protein aggregates, mitochondrial dysfunction, and defects in protein homeostasis..$^{10,11}$ These disease mechanisms are not mutually exclusive and are often interrelated, leading to a final common pathway of motor neuronal death. Through excessive activation of its receptors, glutamate can induce neuronal cell injury and death via increased intracellular calcium and generation of reactive oxygen species. ${ }^{11}$ Dysfunction of mitochondria can also lead to the generation of free radicals, thus oxidative stress is an important and prominent feature of the disease pathogenesis. ${ }^{13}$

\section{Challenges in amyotrophic lateral sclerosis drug discovery}

Despite numerous clinical trials, there have been only two medications in the past 22 years approved specifically for patients with ALS that directly impact the cause of motor neuron degeneration. Disease variability, the lack of sensitive biomarkers and outcome measures, questionable relevance of pre-clinical models, diagnostic delays, and until recently, clinical trial design that did not take into account the significant disease heterogeneity associated with ALS, are some of the potential reasons given for study failure. ${ }^{14}$ Trials that are wide-ranging in their patient recruitment confer statistical challenges for between-treatment group analyses. ${ }^{14}$ It remains possible that the design of prior clinical trials may have precluded the ability to identify a significant effect.
In the absence of proven clinical, molecular or biochemical biomarkers, identification of homogeneous treatment groups has not been possible. Several recent trials have adopted a cohort enrichment strategy in recruiting patients that might show a selective benefit to a proposed treatment mechanism or given a limited trial duration. This strategy has led to the recent approval of edaravone and is being adopted in at least two current clinical trials: NP001 (NCT02794857; Neuraltus Pharmaceuticals, Inc.) and AMX0035 (NCT03127514; Amylyx Pharmaceuticals, Inc.).

\section{Approved treatment options for patients with amyotrophic lateral sclerosis}

In 1995, riluzole (Rilutek ${ }^{\circledR}$, Sanofi-Aventis), an inhibitor of glutamate release and voltage-dependent sodium channels, was approved for the treatment of ALS after demonstrating improved patient tracheotomyfree survival by 2-3 months in two randomized, double-blind, placebocontrolled clinical studies. ${ }^{15-17,18}$

In May 2017, the Food and Drug Administration (FDA) approved edaravone (Radicava ${ }^{\circledR}$, Mitsubishi Tanabe Pharma America, Inc.) for the treatment of ALS. The biochemical properties of edaravone and evidence from in vitro and in vivo studies suggest that it may have protective effects against oxidative stress. Edaravone is thought to be a free radical scavenger that eliminates lipid peroxides and hydroxyl radicals thereby mitigating oxidative injury in central nervous system neurons..$^{19,20}$ Edaravone is administered as a $60 \mathrm{mg}$ intravenous infusion over 60 minutes every day for the first 14 days of treatment, followed by a 14-day drug-free period. Subsequent treatment cycles involve daily dosing for 10 days out of a 14-day period, following by 14-day drug-free periods - termed a 2-weekly dosing schedule. ${ }^{21}$

In addition to pharmacological treatment, there are other major management issues that need to be considered when caring for patients with ALS. In a practice parameter published by the Quality Standards committee of the American Academy of Neurology, it was determined that attendance at specialized multidisciplinary ALS clinics improve patient survival, and effective symptomatic management can enhance patient quality of life. ${ }^{22}$ As such, these form the key recommendations for ALS management by the committee.

\section{Edaravone clinical development program}

The edaravone clinical development program for ALS was conducted in Japan with over 380 patients. An overview of studies is provided in Table 1. Edaravone was initially developed and approved in Japan for the treatment of ischemic stroke. ${ }^{23,24}$ Edaravone also demonstrated efficacy in pre-clinical models of ALS. In wobbler mice, a sporadic ALS-like model, edaravone or vehicle was administered in a blind, randomized protocol. Edaravone-treated wobbler mice demonstrated attenuated forelimb deformity and muscle weakness compared with vehicle-treated mice. ${ }^{25}$ Furthermore, muscle weight, muscle fiber diameter and number of spinal motor neurons were all significantly greater in the edaravone-treated mice compared with vehicletreated mice. ${ }^{25}$ In the SOD-1 transgenic model of ALS, edaravone-treated male rats demonstrated significantly better performance in the landing footsplay functional test. ${ }^{26}$ Additionally, in the SOD-1 transgenic mouse model for ALS, animals treated in a randomized, blind protocol with edaravone demonstrated superior motor performance, a reduction in weight loss, superior grip strength, and significant motor neuron preservation compared with vehicle-treated mice. ${ }^{27}$ After showing promising results in pre-clinical studies, ${ }^{25-27}$ clinical trials of edaravone in ALS were initiated. ${ }^{20}$ 
Table 1: An overview of the edaravone clinical development program in amyotrophic lateral sclerosis

\begin{tabular}{|c|c|c|c|c|}
\hline Trial & Trial type & Patient population & Duration & Summary \\
\hline Study $12^{31}$ & Open-label, phase 2 & $\begin{array}{l}\text { - } 20 \text { patients } \\
\text { - } 30 \text { mg versus } 60 \text { mg edaravone } \\
\text { - } \text { Diagnosis of sporadic or familial ALS }\end{array}$ & 24 weeks & $\begin{array}{l}\text { Edaravone was well-tolerated with } \\
\text { a favorable safety profile. Study met } \\
\text { primary and secondary endpoint; } \\
\text { significantly less decline in ALSFRS-R } \\
\text { and reduction of 3NT levels }\end{array}$ \\
\hline Study $16^{32}$ & $\begin{array}{l}\text { Double-blind, } \\
\text { placebo-controlled } \\
\text { RCT, phase } 3\end{array}$ & $\begin{array}{l}\text { - } 206 \text { patients } \\
\text { - Diagnosis of definite, probable or } \\
\text { probable laboratory-supported ALS } \\
\text { - } F V C \geq 70 \% \\
\text { - } \text { Placebo versus } 60 \mathrm{mg} \text { edaravone }\end{array}$ & $\begin{array}{l}\text { 12-week observation period followed } \\
\text { by 24-week treatment period }\end{array}$ & $\begin{array}{l}\text { No statistically significant difference in } \\
\text { ALSFRS-R score between edaravone } \\
\text { and placebo }\end{array}$ \\
\hline Study $17^{35}$ & $\begin{array}{l}\text { Placebo-controlled } \\
\text { RCT extension of } \\
\text { Study } 16\end{array}$ & $\begin{array}{l}\text { - } 181 \text { patients } \\
\text { Patients who received edaravone in } \\
\text { study } 16 \text { were reassigned to either } \\
\text { edaravone or placebo, followed by } \\
\text { open-label edaravone }\end{array}$ & $\begin{array}{l}24 \text { weeks followed by } 12 \text {-week open-label } \\
\text { edaravone }\end{array}$ & $\begin{array}{l}\text { Beneficial trend favoring edaravone } \\
\text { (ALSFRS-R) but not statistically } \\
\text { significant }\end{array}$ \\
\hline Study $18^{34}$ & $\begin{array}{l}\text { Placebo-controlled } \\
\text { exploratory study }\end{array}$ & $\begin{array}{l}\text { - } 25 \text { patients with more severe ALS } \\
\text { (grade } 3 \text { Japan ALS severity } \\
\text { classification) } \\
\text { - } \text { FVC } \geq 60 \% \\
\text { - } \text { Not statistically powered }\end{array}$ & $\begin{array}{l}\text { 12-week observation period followed } \\
\text { by 24-week treatment period }\end{array}$ & $\begin{array}{l}\text { No difference between edaravone and } \\
\text { placebo in ALSFRS-R score }\end{array}$ \\
\hline Study $19^{36}$ & $\begin{array}{l}\text { Double-blind, } \\
\text { placebo-controlled } \\
\text { RCT, phase } 3\end{array}$ & $\begin{array}{l}\text { - } 137 \text { patients } \\
\text { - } \text { Diagnosis of definite or probable ALS } \\
\text { - } \text { Score of } \geq 2 \text { on all } 12 \text { items of the } \\
\text { ALSFRS-R } \\
\text { - } F V C \geq 80 \% \\
\text { - } \text { Disease duration of } \leq 2 \text { years } \\
\text { - } \text { Placebo versus } 60 \mathrm{mg} \text { edaravone }\end{array}$ & $\begin{array}{l}\text { 12-week observation period followed } \\
\text { by } 24 \text { weeks of treatment, plus a 24-week } \\
\text { open-label extension }\end{array}$ & $\begin{array}{l}\text { Met primary endpoint and multiple } \\
\text { secondary endpoints; statistically } \\
\text { reduced functional progression as } \\
\text { measured by the ALSFRS-R }\end{array}$ \\
\hline
\end{tabular}

3NT = 3-nitrotyrosine; $A L S$ = amyotrophic lateral sclerosis; ALSFRS-R = Revised ALS Functional Rating Scale; FVC = forced vital capacity; $R C T=$ randomized controlled trial.

The ALS Functional Rating Scale-Revised (ALSFRS-R) is a validated rating instrument often used in clinical trials to monitor the progression of disability in patients with ALS. ${ }^{28}$ It is a 12-item scale across four domains: bulbar, fine motor, gross motor and respiratory (see Table 2). ${ }^{29}$ The ALSFRS-R was used as a primary endpoint throughout all the studies in the edaravone clinical development program. The ALSFRS-R is widely used due to its ease of use and correlation with disease status and level of disability. ${ }^{28}$ However, the ALSFRS-R is relatively insensitive to progression over short periods of time, may have a floor effect in advanced disease, and since it is a subjective test, it may be affected by a patient's mood, i.e., depression or optimism. ${ }^{30}$

The first trial to investigate the safety and efficacy of edaravone in patients with ALS was MCl186-12, a phase 2 open-label study. Patients received either $30 \mathrm{mg}(n=5)$ or $60 \mathrm{mg}(n=15)$ edaravone in a 2-weekly dosing schedule, for six cycles of treatment (a total of 6 months). Inclusion criteria were a diagnosis of familial or sporadic ALS. Exclusion criteria included tracheotomy, artificial respiration, dyspnea, or a stable ALSFRS-R. The primary endpoint was the change in the ALSFRS-R during the 6-month edaravone treatment period, compared with the change in ALSFRS-R during the natural course of ALS in the 6 months prior to treatment. Secondary endpoints included the change in cerebrospinal fluid (CSF) 3-nitrotyrosine (3NT; a marker of oxidative stress) levels at the end of the treatment period. Safety assessments included the incidence of adverse events. ${ }^{31}$ The decline in ALSFRS-R score was significantly less during the 6-month treatment period with $60 \mathrm{mg}$ edaravone, compared with the 6-month period prior to edaravone administration $(2.3 \pm 3.6$ points versus $4.7 \pm 2.1$ points respectively; $p=0.039$ ). A marked reduction in the level of CSF 3NT to almost undetectable levels was observed in almost all patients receiving $60 \mathrm{mg}$ edaravone, suggesting that neuronal cells were being protected from oxidative stress. Edaravone had a favorable safety profile. No clinically significant adverse drug reactions were observed and edaravone was well tolerated in this study. ${ }^{31}$

Study MCl186-16 was a phase 3, double-blind, parallel-group, placebocontrolled trial investigating the efficacy and safety of edaravone in patients with ALS, consisting of a 12-week observation period followed by a 24-week randomized treatment period. The study was carried out across 29 different sites in Japan. The primary efficacy endpoint was the change in the ALSFRS-R during the 24-week treatment period. Secondary endpoints included changes in percentage forced vital capacity (FVC), the Modified Norris Scale Score (an alternative rating scale for ALS that assesses limb and bulbar function), grip strength (left/right mean), pinch strength (left/right mean), and time to death. Safety assessments included the incidence and severity of adverse events. ${ }^{32}$ Inclusion criteria included individuals aged 20-75 years with a diagnosis of definite, probable or probable laboratory-supported ALS (according to the El Escorial and revised Airlie House diagnostic criteria), FVC of $\geq 70 \%$, disease duration $<3$ years, and a change in the ALSFRS-R during the 12-week observation period of -1 to -4 points. Patients had a Japanese ALS severity classification of 1 or 2 (see Table 3). Administration regimen of riluzole 


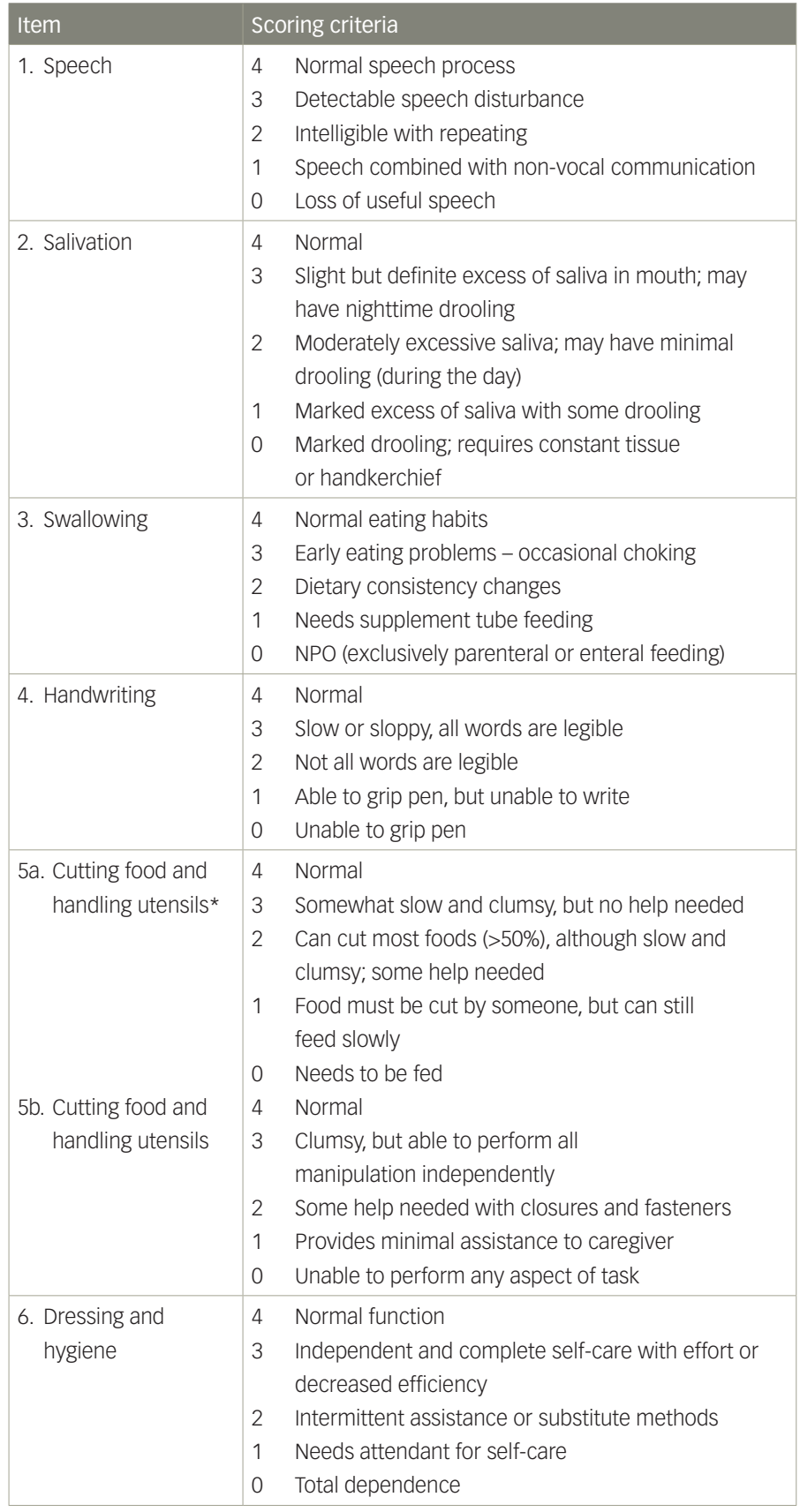

was required not to be changed during the study (88.5\% of patients in the placebo group and $89.1 \%$ of patients in the edaravone group were taking riluzole). Exclusion criteria included reduced respiratory function or dyspnea, and complications preventing drug efficacy evaluation or those requiring hospitalization. ${ }^{32}$

Changes in the ALSFRS-R (least-squares [LS] mean \pm standard error [SE]) during the 24-week treatment were numerically smaller in the edaravone group $(-5.70 \pm 0.85 ; n=100)$ compared with the placebo group $(-6.35 \pm 0.84 ; n=99)$ but were not statistically significant (intergroup difference $0.65 \pm 0.78 ; p=0.411$ ). There were no significant differences between edaravone treatment and placebo for any of the

\begin{tabular}{|c|c|}
\hline em & Scor \\
\hline $\begin{array}{l}\text { 7. Turning in bed } \\
\text { and adjusting } \\
\text { bed clothes }\end{array}$ & $\begin{array}{ll}4 & \text { Normal function } \\
3 & \text { Somewhat slow and clumsy but no help needed } \\
2 & \text { Can turn alone, or adjust sheets, but with } \\
& \text { great difficulty } \\
1 & \text { Can initiate, but not turn or adjust sheets alone } \\
0 & \text { Helpless }\end{array}$ \\
\hline 8. Walking & $\begin{array}{ll}4 & \text { Normal } \\
3 & \text { Early ambulation difficulties } \\
2 & \text { Walks with assistance } \\
1 & \text { Non-ambulatory functional movement } \\
0 & \text { No purposeful leg movement }\end{array}$ \\
\hline 9. Climbing stairs & $\begin{array}{ll}4 & \text { Normal } \\
3 & \text { Slow } \\
2 & \text { Mild unsteadiness or fatigue } \\
1 & \text { Needs assistance } \\
0 & \text { Cannot do }\end{array}$ \\
\hline 10. Dyspnea & $\begin{array}{ll}4 & \text { None } \\
3 & \text { Occurs when walking } \\
2 & \text { Occurs with one or more of the following: eating, } \\
\text { bathing, dressing } \\
1 & \begin{array}{l}\text { Occurs at rest: difficulty breathing when either sitting } \\
\text { or lying }\end{array} \\
0 & \begin{array}{l}\text { Significant difficulty: considering using mechanical } \\
\text { respiratory support }\end{array}\end{array}$ \\
\hline 11. Orthopnea & $\begin{array}{ll}4 & \text { None } \\
3 & \begin{array}{l}\text { Some difficulty sleeping at night due to shortness } \\
\text { of breath, does not routinely use more than }\end{array} \\
\text { two pillows } \\
2 & \begin{array}{l}\text { Needs extra pillows in order to sleep (more } \\
\text { than two) }\end{array} \\
1 & \text { Can only sleep sitting up } \\
0 & \text { Unable to sleep without mechanical assistance }\end{array}$ \\
\hline $\begin{array}{l}\text { 12. Respiratory } \\
\text { insufficiency }\end{array}$ & $\begin{array}{ll}4 & \text { None } \\
3 & \text { Intermittent use of BiPAP } \\
2 & \text { Continuous use of BiPAP during the night } \\
1 & \text { Continuous use of BiPAP during the day and night } \\
0 & \text { Invasive mechanical ventilation by intubation } \\
& \text { or tracheotomy }\end{array}$ \\
\hline
\end{tabular}

*Patients without G-tube - use $5 \mathrm{~b}$ if $>50 \%$ is though G-tube.

**Patients with $G$-tube - $5 b$ is used if the patient has a G-tube and only if it is the primary method (>50\%) of eating.

$A D L=$ activities of daily living; BiPAP = Bilevel Positive Airway Pressure;

$G$-tube = gastrostomy tube; $N P O=$ nothing by mouth .

Reproduced with permission from Cedarbaum et al., 1999.28

secondary outcomes except pinch strength (analyzed by repeated measures analysis of variance), which showed statistically significant difference favoring edaravone $(p=0.038)$. Frequency and severity of reported adverse events were similar in the two groups. Adverse events and serious adverse events occurred in $88.5 \%$ and $23.1 \%$ of patients in the placebo group respectively, and $89.2 \%$ and $17.6 \%$ of patients treated with edaravone respectively. No serious adverse drug reactions occurred in either treatment group. ${ }^{32} \mathrm{~A}$ stratified post-hoc analysis by diagnostic category revealed that the change in ALSFRS-R score was greater in those patients fulfilling the El Escorial and revised Airlie House diagnostic criteria for clinically definite ALS: -8.7 in the placebo group versus -6.7 in the edaravone group. ${ }^{32,23}$ 
Table 3: Japanese amyotrophic lateral sclerosis severity scale ${ }^{32}$

\begin{tabular}{|c|c|}
\hline Grade & Signs and symptoms \\
\hline 1 & Able to work or perform housework \\
\hline 2 & Independent living but unable to work \\
\hline 3 & Requiring assistance for eating, excretion, or ambulation \\
\hline 4 & $\begin{array}{l}\text { Presence of respiratory insufficiency, difficulty in coughing out sputum, } \\
\text { or dysphagia }\end{array}$ \\
\hline 5 & $\begin{array}{l}\text { Using a tracheostomy tube, tube feeding, or tracheostomy positive- } \\
\text { pressure ventilation }\end{array}$ \\
\hline
\end{tabular}

\section{Rationale for cohort enrichment}

The lack of significance in the primary efficacy endpoint between the placebo and treatment arm during study MCl186-16 was investigated in a follow-up analysis. This revealed that $25 \%$ of patients in the edaravone group and $26 \%$ of patients in the placebo group showed a change of 0 or -1 point in ALSFRS-R score indicating a more slowly progressive form of the disease than had originally been anticipated when the trial was designed, and thus attenuating the power of the study. ${ }^{32}$ Therefore, it was of interest to identify a group of patients to include in a clinical study who would show adequate progression.

In an exploratory study (MCl186-18) of edaravone or placebo in 25 patients with severe ALS (Japan ALS severity classification of grade 3), there was no difference in ALSFRS-R between the treatment groups. The study was not powered to statistically evaluate the efficacy of edaravone, and therefore the effect of edaravone on patients with more severe ALS is inconclusive. ${ }^{34}$ Patients with more advanced disease may have already decreased several points on the ALSFRS-R scale to 0 or 1 , therefore assessments of further changes are difficult. Due to these potential floor effects of the ALSFRS-R in patients with more severe ALS, this study provided a rationale as to why patients with severe ALS were not included in the proposed enriched cohort of patients. To detect changes in ALSFRS-R in a clinical study it is important to start with patients that are less functionally impaired at baseline, e.g. those with individual item scores of 2 or more. ${ }^{33}$

Post-hoc analyses of the MCl186-16 study were performed in an attempt to identify a group of patients that when using the ALSFRS-R in a 6-month clinical study, could elucidate a treatment effect. Two patient subgroups were defined from the post-hoc analyses: Step 1 - the efficacy-expected subpopulation (EESP) with FVC of $\geq 80 \%$ before treatment and $\geq 2$ points for all item scores in the ALSFRS-R at baseline; and Step 2 - the greater-efficacyexpected subpopulation within the EESP, (dpEESP2y) with a diagnosis of 'definite' or 'probable' ALS according to the El Escorial and revised Airlie House diagnostic criteria, and within 2 years of initial ALS symptom onset at the time of giving informed consent. ${ }^{33}$ A comparison of the subgroup characteristics and the full analysis set is shown in Table 4. Coordinating investigators and medical expert consensus were responsible for setting these subgroup criteria. ${ }^{33}$

Changes in the ALSFRS-R (LS mean) during the 24-week treatment in the EESP subgroup were -7.06 for placebo $(n=46)$ and -4.85 for edaravone $(n=53)$, with a significant intergroup difference of $2.20 \pm 1.03$ ( $p=0.0360)$. Changes in the ALSFRS-R during the 24-week treatment in the dpEESP2y subgroup were -7.59 for placebo $(n=29)$ and -4.58 for edaravone $(n=39)$, with a significant intergroup difference of $3.01 \pm 1.33(p=0.0270){ }^{33}$ These results suggested that edaravone slowed the progression of ALS in the two defined subpopulations when compared with placebo. Additional efficacy was demonstrated when the same analyses were carried out for the secondary efficacy endpoints. Changes in percentage of FVC (LS mean) during the 24-week treatment in the EESP subgroup were -16.35 for placebo and -11.74 for edaravone, with a significant intergroup difference $(p=0.0488)$. Changes in percentage of FVC during the 24-week treatment in the dpEESP2y subgroup were -19.69 for placebo and -13.40 for edaravone, with a significant intergroup difference $(p=0.0467)$. Similar significant changes were also observed in the Modified Norris Scale scores for the two specified subgroups.

Further efficacy of longer-term edaravone was explored in study MCI186-17, a randomized 24-week extension to study MCl186-16. The results suggest that edaravone might have potential efficacy for up to 15 cycles when used to treat patients with ALS. Furthermore, post-hoc analyses of this study for both the EESP and dpEESP2y populations showed beneficial trends favoring edaravone. ${ }^{35}$ Therefore, it was proposed that efficacy of edaravone should be demonstrated in a second prospective phase 3 study if the cohort of patients was enriched according to the dpEESP2y criteria. ${ }^{33}$

\section{Strategic clinical study design through patient population enrichment}

Study MCl186-19 was a phase 3, double-blind, randomized, parallel-group, placebo-controlled trial investigating the efficacy and safety of edaravone in the well-defined post-hoc step 2 (dpEESP2y) population of patients with ALS. ${ }^{36}$ The study was carried out with 137 patients recruited from 31 hospitals in Japan. Inclusion criteria were individuals aged $20-75$ years with a diagnosis of definite or probable ALS according to the El Escorial and revised Airlie House criteria, a Japanese ALS severity classification of 1 or 2 , and a decrease in the ALSFRS-R score of 1-4 during a 12-week observation period. Based on the post-hoc subgroup analysis, eligible patients also had scores of $\geq 2$ points for all item scores in the ALSFRS-R, a FVC $\geq 80 \%$, and duration of disease from the first ALS symptom of $\leq 2$ years. Exclusion criteria included a score of $\leq 3$ of ALSFRS-R items for dyspnea, orthopnea or respiratory insufficiency. Patients who had already been given riluzole could continue to receive riluzole provided that the regimen remained unchanged, but initiation of riluzole after the start of the observation period was prohibited. Riluzole use was observed in $91 \%$ of patients in both treatment groups. ${ }^{36}$ These steps were taken to manage variability in the patient population and ensure the inclusion of patients with progressive disease, and at the same time excluding patients with too slowly or rapidly progressing disease..$^{37}$ Following the 12-week observation period, eligible patients were randomized 1:1 to placebo or edaravone. The primary efficacy endpoint was a change in ALSFRS-R score from baseline to week 24, following six cycles of treatment. Secondary endpoints included change in percentage of FVC, Modified Norris Scale scores and grip and pinch strengths. Safety endpoints included the incidence of adverse events. ${ }^{36}$

Changes in the ALSFRS-R (LS mean change \pm SE) through the 24-week study period were $-5.01 \pm 0.64$ in the edaravone group ( $n=68)$, compared with $-7.50 \pm 0.66$ in the placebo group $(n=66)$. The intergroup comparison revealed a statistically significant difference $(2.49 \pm 0.76 ; p=0.0013)$ (Figure 1). This translates into a 33\% difference between the placebo and edaravone group. It should be noted that in a survey of the Northeast ALS Consortium, a 25\% change in the ALSFRS-R score was considered clinically meaningful. ${ }^{38}$ Efficacy was also demonstrated in some of the secondary endpoints including the total Modified Norris Scale score with 
Table 4: Comparison of analysis groups in a post-hoc subgroup analysis of the MCl186-16 study ${ }^{33}$

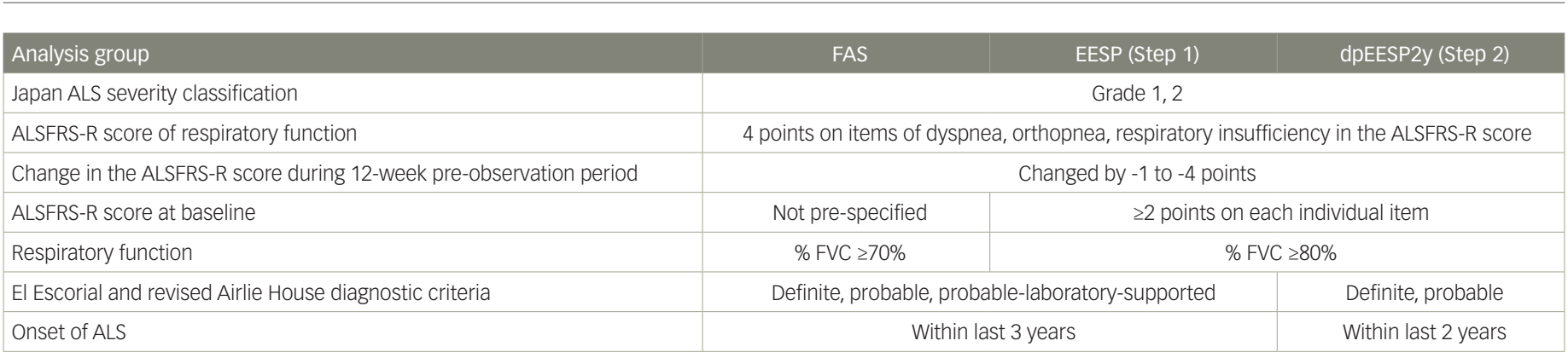

ALS = amyotrophic lateral sclerosis; ASLFRS-R = revised ALS Functional Rating Scale; dpEESP2y = subgroup of the EESP, containing patients with a diagnosis of 'definite' or 'probable' ALS according to the El ESCorial and revised Airlie House diagnostic criteria and with disease duration of $\leq 2$ years; EESP = efficacy-expected subpopulation of patients with ALS (\% FVC of $\geq 80 \%$ before treatment and $\geq 2$ points for all item scores in the ALSFRS- $R$ before treatment; FAS = full analysis set; \% FVC = percentage forced vital capacity.

a LS mean change \pm SE through the 24-week study period of $-15.91 \pm 1.97$ in the edaravone group, compared with $-20.80 \pm 2.06$ in the placebo group (intergroup difference $4.89 \pm 2.35 ; \mathrm{p}=0.0393$ ). The secondary endpoint which assessed quality of life, ALSAQ-40, was statistically significant in favor of edaravone compared with placebo (LS mean \pm SE was $-8.79 \pm 4.03$, $\mathrm{p}=0.031$ ). Changes in the percentage of FVC and grip and pinch strengths showed a tendency towards improvement with edaravone but were not statistically significant. The frequency of adverse events was comparable between the two treatment groups, and no serious adverse drug reactions were reported in either group. ${ }^{36}$

In an open-label 24-week extension of the trial, 65 patients who had received edaravone in the double-blind study period remained on treatment and 58 patients who received placebo switched to edaravone. After a total of 48 weeks, the mean change in the ALSFRS-R score was - 8.0 in patients who continued receiving edaravone and -10.9 in those who switched to edaravone from the placebo arm. ${ }^{39}$ Whilst further evaluation of long-term treatment with edaravone is needed, this study extension provides a rationale for early treatment with edaravone since patients continuing on edaravone appeared to be progressing at a slower rate than those who had recently switched.

\section{Lessons for future clinical trials}

Approval of edaravone in the US has marked a new chapter in pharmaceutical development for several reasons. Firstly, because it was the first case of an FDA approval that was granted based on clinical trials that had a smaller patient number than most that have preceded it; secondly, because the key trials for edaravone were performed outside the US, and finally because of the novel strategic clinical trial design involving cohort enrichment. This raises hope and interest that other drugs may be approved in a similar fashion in the future, particularly for neurodegenerative diseases where there is still a large unmet medical need.

Study MCl186-19 highlights a new approach to ALS clinical trials that could be applicable to studies in other neurodegenerative diseases. A key factor contributing to the failure of past clinical trials in ALS is the study design; in particular the variability in disease presentation and progression. ${ }^{14}$ The syndromic nature of ALS encompasses a very heterogeneous group of motor neuron disease(s), as observed in the MCl186-16 study where $26 \%$ of placebo patients and $25 \%$ of edaravone patients were defined as slow progressors. ${ }^{32}$ This suggests that within the population of patients with ALS there may be several different patient categories of disease progression. When studying a drug that slows disease progression over
Figure 1: Study MCI186-19 primary efficacy endpointchange in ALSFRS-R in edaravone and placebo treatment groups

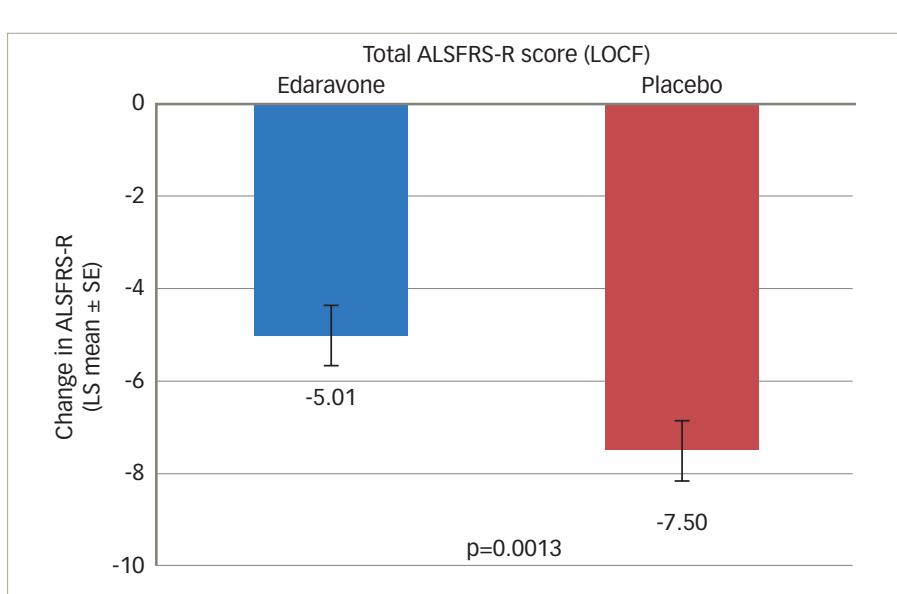

A statistically significant 33\% reduction in the expected decline in the ALSFRS-R score was detected at the end of the 6-month trial period. At baseline, the average ALSFRS- $R$ score in both treatment groups was 42 (where 48 is a perfect score; Table 2). Over 6 months, patients in the edaravone group averaged a 5.0-point reduction on the ALSFRS- $R$, compared with a 7.5-point reduction for patients in the placebo group. The between-group difference in adjusted mean (LS mean \pm SE [95\% CI]) was $2.49 \pm 0.76$ $(0.99,3.98)$. For placebo, $n=66$ and for edaravone, $n=68$. Figure adapted from Edaravone (MCl-186) ALS 19 Study Writing Group, 2017. ${ }^{36} \mathrm{ALS}=$ amyotrophic lateral sclerosis; ALSFRS-R = Revised ALS Functional Rating Scale; $\mathrm{Cl}=$ confidence interval;

$L O C F=$ last observation carried forward; $L S=$ least square; $S E=$ standard error.

a finite trial duration, it is difficult to elucidate a treatment effect when comparing study arms with large numbers of patients who are not progressing. By defining a very specific patient population in the study MCl186-16 post-hoc analysis, and subsequent study MCl186-19, many of these 'non-progressing' patients were eliminated.

It is important to note that the lack of the expected progression in the placebo group in study MCl186-16 led to the cohort enrichment in study MCI186-19, and ultimately the detection of a significant treatment effect. However, we can postulate that had the placebo group in study MCl186-16 behaved like historical controls, it is likely this study would have met the primary end point as well. As such, it is possible that edaravone does not necessarily work selectively in rapidly progressing patients. Future research incorporating relevant biomarkers may help elucidate better edaravone's mechanism of action, pharmacodynamic considerations, and subsequently the ALS phenotypes that may benefit preferentially from treatment. 
Post-hoc analyses of previous randomized control trials have identified responder groups that might have been overlooked, providing possible reasons for study failure. ${ }^{40}$ Without the follow-up analyses and subsequent clinical study with edaravone, efficacy would never have been demonstrated and approval of the drug would not have been realized. ${ }^{41}$ By specifying a well-defined patient population, investigators can address some of the phenotypic variability observed in patients with $\mathrm{ALS}^{40}$ while minimizing patient risk, study duration, and sample size to improve efficiency and promote statistical power. ${ }^{42}$ The concept of cohort enrichment is particularly important if the enrichment criteria are related to the hypothesized mechanism of action; while this was not the case for study MCl186-19, it could be relevant for future clinical studies in ALS.

Another potential problem revealed in the design of past clinical trials is the expected treatment effects, sometimes $40-50 \%$ greater than placebo, which are often unrealistic. ${ }^{40}$ The difference in ALSFRS-R in the MCl186-19 study equates to $33 \%$ less functional loss in the edaravone group compared with the placebo group. The impact of slower functional decline for patients could mean an extension in their ability to independently perform activities of daily living, and less deterioration in their walking, climbing or swallowing abilities. such effects are very important and significant for patients living with ALS.

\section{Conclusion}

Cohort enrichment strategies, coupled with a relevant biomarker consistent with the expected action of the drug, should now be considered as a viable and perhaps preferred design for future studies. It is imperative that future clinical trials in ALS and other neurodegenerative diseases accommodate innovative design modifications. By doing so, it is hoped that edaravone will be the first of many new disease-modifying treatments to be approved over the coming years. $\square$
1. Riva N, Agosta F, Lunetta $\mathrm{C}$, et al. Recent advances in amyotrophic lateral sclerosis. J Neurol. 2016;263:1241-54.

2. Swinnen $B$, Robberecht $W$. The phenotypic variability of amyotrophic lateral sclerosis. Nat Rev Neurol. 2014;10:661-70.

3. Jenkins TM, Hollinger H, McDermott CJ. The evidence for symptomatic treatments in amyotrophic lateral sclerosis. Curr Opin Neurol. 2014;27:524-31.

4. Gibbons C, Pagnini F, Friede T, et al. Treatment of fatigue in amyotrophic lateral sclerosis/motor neuron disease. cochrane Database Syst Rev. 2018:1:CD011005

5. Turner MR, Hardiman O, Benatar M, et al. Controversies and priorities in amyotrophic lateral sclerosis. Lancet Neurol. 2013;12:310-22.

6. Paganoni S, Macklin EA, Lee A, et al. Diagnostic timelines and delays in diagnosing amyotrophic lateral sclerosis (ALS). Amyotroph Lateral Scler Frontotemporal Degener. 2014;15:453-6.

7. Van Damme P, Robberecht W, Van Den Bosch L. Modelling amyotrophic lateral sclerosis: progress and possibilities. Dis Model Mech. 2017; 10:537-49.

8. Turner MR, Al-Chalabi A, Chio A, et al. Genetic screening in sporadic ALS and FTD. I Neurol Neurosurg PSychiatry. 2017;88:1042-4.

9. Mehta P, Antao V, Kaye W, et al. Prevalence of amyotrophic latera sclerosis - United States, 2010-2011. MMWR Suppl. 2014:63:1-14.

10. Brown RH, Al-Chalabi A. Amyotrophic lateral sclerosis. N Eng/ J Med. 2017:377:162-72.

11. Kiernan $M C$, vucic $S$, Cheah $B C$, et al. Amyotrophic lateral sclerosis. Lancet. 2011;377:942-55.

12. Miller RG, Appel SH. Introduction to supplement: the current status of treatment for ALS. Amyotroph Lateral Scler Frontotemporal Degener. 2017;18:1-4.

13. Veyrat-Durebex C, Corcia P, Piver E, et al. Disruption of TCA cycle and glutamate metabolism identified by metabolomics in an in vitro model of amyotrophic lateral sclerosis. Mol Neurobiol. in vitro model of amy

14. Katyal N, Govindarajan R. Shortcomings in the current amyotrophic lateral sclerosis trials and potential solutions for improvement. Front Neurol. 2017:8:521.

15. Covis Pharmaceuticals Inc. Rilutek prescribing information 2016. Available at: www.accessdata.fda.gov/drugsatfda_docs/ label/2016/020599s017/bl.pdf (accessed November 30, 2017).

16. Lacomblez L, Bensimon G, Leigh PN, et al. Dose-ranging study of riluzole in amyotrophic lateral sclerosis. Amyotrophic Lateral Sclerosis/Riluzole Study Group II. Lancet. 1996;347:1425-31.

17. Bensimon $G$, Lacomblez $L$, Meininger V. A controlled trial of riluzole in amyotrophic lateral sclerosis. ALS/Riluzole Study Group N Eng/ J Med. 1994;330:585-91.
18. Miller RG, Mitchell JD, Moore DH. Riluzole for amyotrophic lateral sclerosis (ALS)/motor neuron disease (MND). Cochrane Database Syst Rev. 2012;3:CD001447.

19. Watanabe T, Yuki S, Egawa M, Nishi H. Protective effects of $\mathrm{MCl}-186$ on cerebral ischemia: possible involvement of free radical scavenging and antioxidant actions. J Pharmacol Exp Ther. 1994;268:1597-604.

20. Yamamoto T, Yuki S, Watanabe T, et al. Delayed neuronal death prevented by inhibition of increased hydroxyl radical formation in a transient cerebral ischemia. Brain Res. 1997:762:240-2.

21. Mitsubishi Tanabe Pharma Corporation. Radicava prescribing information. 2017. Available at: www.accessdata.fda.gov/drugsatfda docs/label/2017/209176lbl.pdf (accessed November 30, 2017).

22. Miller RG, Jackson CE, Kasarskis EJ, et al. Practice paramete update: the care of the patient with amyotrophic lateral sclerosis: multidisciplinary care, symptom management, and cognitive/ behavioral impairment (an evidence-based review): report of the Quality Standards Subcommittee of the American Academy of Neurology. Neurology. 2009;73:1227-33.

23. Edaravone Acute Infarction Study Group. Effect of a novel free radical scavenger, edaravone (MCl-186), on acute brain infarction. Randomized, placebo-controlled, double-blind study at multicenters. Cerebrovasc Dis. 2003:15:222-9.

24. Houkin K, Nakayama N, Kamada K, et al. Neuroprotective effect of the free radical scavenger $\mathrm{MCl}-186$ in patients with cerebral infarction: clinical evaluation using magnetic resonance imaging and spectroscopy. J Stroke Cerebrovasc Dis. 1998;7:315-22.

25. Ikeda K, Iwasaki Y. Edaravone, a free radical scavenger, delayed symptomatic and pathological progression of motor neuron disease in the wobbler mouse. PLOS One. 2015;10:e0140316.

26. Aoki M, Warita $\mathrm{H}$, Mizuno H, et al. Feasibility study for functional test battery of SOD transgenic rat (H46R) and evaluation of edaravone, a free radical scavenger. Brain Res. 2011;1382:321-5

27. Ito $\mathrm{H}$, Wate $\mathrm{R}$, Zhang J, et al. Treatment with edaravone, initiated at symptom onset, slows motor decline and decreases SOD1 deposition in ALS mice. Exp Neurol. 2008;213:448-55.

28. Cedarbaum JM, Stambler N, Malta E, et al. The ALSFRS-R: a revised ALS functional rating scale that incorporates assessments of respiratory function. BDNF ALS Study Group (Phase III). J Neuro Sci. 1999;169:13-21.

29. European Network to Cure ALS (ENCALS). ALS Functional Rating Scale Revised (ALS-FRS-R). 2015. Available at: www.encals.eu/wpcontent/uploads/2016/09/ALS-Functional-Rating-Scale-Revisedfill-in-form.pdf (accessed March 13, 2018)

30. Rutkove SB. Clinical measures of disease progression in amyotrophic lateral sclerosis. Neurotherapeutics. 2015;12:384-93.
31. Yoshino $\mathrm{H}$, Kimura A. Investigation of the therapeutic effects of edaravone, a free radical scavenger, on amyotrophic lateral sclerosis (Phase II study). Amyotroph Lateral Scler. 2006;7:241-5.

32. Abe K, Itoyama Y, Sobue G, et al. Confirmatory double-blind, parallel-group, placebo-controlled study of efficacy and safety of edaravone (MCl-186) in amyotrophic lateral sclerosis patients. Amyotroph Lateral Scler Frontotemporal Degener. 2014;15:610-7.

33. Edaravone (MCl-186) ALS 16 Study Group. A post-hoc subgroup analysis of outcomes in the first phase III clinical study of edaravone (MCl-186) in amyotrophic lateral sclerosis. Amyotroph Lateral Scler Frontotemporal Degener. 2017;18:11-9.

34. Writing Group on behalf of the Edaravone (MCl-186) ALS 18 Study Group. Exploratory double-blind, parallel-group, placebocontrolled study of edaravone (MCl-186) in amyotrophic lateral sclerosis (Japan ALS severity classification: Grade 3, requiring assistance for eating, excretion or ambulation). Amyotroph Lateral Scler Frontotemporal Degener. 2017:18:40-8.

35. Writing Group on behalf of the Edaravone (MCl-186) ALS 17 Study Group. Exploratory double-blind, parallel-group, placebo-controlled extension study of edaravone (MCl-186) in amyotrophic lateral sclerosis. Amyotroph Lateral Scler Frontotemporal Degener. 2017;18:20-31.

36. Writing Group on behalf of the Edaravone (MCl-186) ALS 19 Study Group. Safety and efficacy of edaravone in well defined patients with amyotrophic lateral sclerosis: a randomised, double-blind, placebo-controlled trial. Lancet Neurol. 2017;16:505-12.

37. Akimoto M, Nakamura $K$ for the Writing Group on behalf of the Edaravone (MCl-186) ALS 19 Study Group. Edaravone for treatment of early-stage ALS - Authors' reply. Lancet Neurol. 2017; $16: 772$

38. Castrillo-Viguera C, Grasso DL, Simpson E, et al. Clinical significance in the change of decline in ALSFRS-R. Amyotroph Lateral Scler. 2010:11:178-80.

39. Writing Group on behalf of the Edaravone (MCl-186) ALS 19 Study Group. Open-label 24-week extension study of edaravone (MCl186) in amyotrophic lateral sclerosis. Amyotroph Lateral Scler Frontotemporal Degener. 2017;18:55--63.

40. Mitsumoto H, Brooks BR, Silani V. Clinical trials in amyotrophic lateral sclerosis: why so many negative trials and how can trials be improved? Lancet Neurol. 2014:13:1127-38.

41. Maragakis NJ. What can we learn from the edaravone development program for ALS? Amyotroph Lateral Scler Frontotemporal Degener. 2017;18:98-103.

42. Nicholson KA, Cudkowicz ME, Berry JD. Clinical trial designs in amyotrophic lateral sclerosis: does one design fit all? Neurotherapeutics. 2015;12:376-83. 